International Journal of Pure and Applied Mathematics

Volume 93 No. 2 2014, 285-295

ISSN: 1311-8080 (printed version); ISSN: 1314-3395 (on-line version)

url: http://www.ijpam.eu

doi: http://dx.doi.org/10.12732/ijpam.v93i2.12

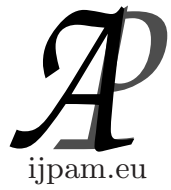

\title{
SOLUTION OF SECOND ORDER LINEAR AND NONLINEAR ORDINARY DIFFERENTIAL EQUATIONS USING LEGENDRE OPERATIONAL MATRIX OF DIFFERENTIATION
}

\author{
Chahn Yong Jung ${ }^{1}$, Zeqing $\mathrm{Liu}^{2}$, Arif Rafiq ${ }^{3}$, Faisal $\mathrm{Ali}^{4}$, Shin Min Kang ${ }^{5} \S$ \\ ${ }^{1}$ Department of Business Administration \\ Gyeongsang National University \\ Jinju, 660-701, KOREA \\ ${ }^{2}$ Department of Mathematics \\ Liaoning Normal University \\ Dalian, Liaoning, 116029, P.R. CHINA \\ ${ }^{3}$ Department of Mathematics \\ Lahore Leads University \\ Lahore, 54810, PAKISTAN \\ ${ }^{4}$ Centre for Advanced Studies in Pure and Applied Mathematics \\ Bahauddin Zakariya University \\ Multan, 60800, PAKISTAN \\ ${ }^{5}$ Department of Mathematics and RINS \\ Gyeongsang National University \\ Jinju 660-701, KOREA
}

\begin{abstract}
Following the approach of [9], in this paper, an approach using Tau method based on Legendre operational matrix of differentiation has been introduced for solving general form of second order linear and nonlinear ordinay differential equations. With the implementation of this scheme the actual problem is converted into a system of algebraic equations, whose solutions are the Legendre coefficients. Some illustrative examples are also given to demonstrate the validity and efficiency of the method.
\end{abstract}

Received: March 10, 2014

(c) 2014 Academic Publications, Ltd. url: www.acadpubl.eu

$\S_{\text {Correspondence author }}$ 
AMS Subject Classification: 65L99

Key Words: Legendre operational matrix of differentiation

\section{Introduction}

Most problems in mathematical physics, engineering, astrophysics and many physical phenomina are governed by differential equations. The exact analytical solutions of such problems, except a few, are difficult to obtain. Many researchers have made attempts to rectify this problem and are able to develop new techniques for obtaining solutions which convincingly approximate the exact solution (see [3], [4], [12], [15] and references therein). Recently, the techniques like variational iteration method [6], Adomion decomposition method [1], [7] and homotopy perturbation method [14] have been exercised by the researchers. Singular initial value problems modeled by second order nonlinear ordinary differential equations have been a scorching topic for various mathematicians and physists during the recent years. Some researchers have studied the special cases of one specific equation in this category, the Lane-Emden type equation

$$
y^{\prime \prime}(x)+\frac{\alpha}{x} y^{\prime}(x)+f(x, y)=g(x), \quad \alpha, x \geq 0
$$

with the initial conditions

$$
y(0)=a \quad \text { and } \quad y^{\prime}(0)=0,
$$

where the prime denotes the differentiation with respect to $x, a$ is a constant, $f(x, y)$ and $g(x)$ are nonlinear continuous functions. Lane-Emden type equation is a nonlinear differential equation which has singularity at the origin and describes the equilibrium density distribution in self-gravitating sphere of polytrophic isothermal gas. This equation is primarily importent in the field of stellar structure, radiative cooling and modeling of clusters of galaxies. It is known that the analytic solution of equation (1.1) is possible [5] in the neighbourhood of its singular point.

In [8], the author considered equation (1.1) taking $\alpha=2, f(x, y)=f(y)=$ $y^{n}, g(x)=0$ and $a=1$, i.e.,

$$
y^{\prime \prime}(x)+\frac{2}{x} y^{\prime}(x)+y^{n}=0, \quad x \geq 0,
$$

which has another form

$$
\frac{1}{x^{2}} \frac{d}{d x}\left(x^{2} \frac{d y}{d x}\right)+y^{n}=0
$$


subject to initial conditions

$$
y(0)=1 \quad \text { and } \quad y^{\prime}(0)=0
$$

Pandey et al. [9] have used Legendre operational matrix of differentiation to solve the same type of equations with $\alpha=2$ and $g(x)=0$. Davis [5] modeled isothermal gas spheres equation (when $\left.f(x, y(x))=e^{y(x)}\right)$.

The aim of this paper is to find the solution of the generalized form of equation (1.1), i.e.,

$$
y^{\prime \prime}(x)+p(x) y^{\prime}(x)+f(x, y)=g(x), \quad \alpha, x \geq 0
$$

with suitable initial conditions. We have applied Tau method based on Legendre operational matrix of differentiation to find the solution of equation (1.6). In Section 3 , we give some examples by taking different values of $p(x), f(x, y)$ and $g(x)$ to illustrate the validity and efficiency of our method.

\section{Legendre Polynomials and its Operational Matrix of Differentiation}

The Legendre polynomials of order $m$ are defined on the interval $[-1,1]$ and are denoted by $L_{m}(t)$. These polynomials can be determined with the help of following recurrence relation

$$
\begin{aligned}
L_{0}(t) & =1, \quad L_{1}(t)=t, \\
L_{m+1}(t) & =\frac{2 m+1}{m+1} t L_{m}(t)-\frac{m}{m+1} L_{m-1}(t), \quad m=1,2, \ldots
\end{aligned}
$$

By taking $t=2 x-1$, the so called shifted Legendre polynomials can be defined on the interval $t \in[0,1]$. Let the shifted Legendre polynomials $L_{m}(2 x-1)$ be denoted by $P_{m}(x)$. Then $P_{m}(x)$ can be obtained as follows

$$
P_{m+1}(x)=\frac{(2 m+1)(2 x-1)}{m+1} P_{m}(x)-\frac{m}{m+1} P_{m-1}(x), \quad m=1,2, \ldots,
$$

where $P_{0}(x)=1$ and $P_{1}(x)=2 x-1$. The analytic form of the shifted Legendre polynomials $P_{m}(x)$ of degree $m$ are given by

$$
P_{m}(x)=\sum_{i=0}^{m}(-1)^{m+i} \frac{(m+i) ! x^{i}}{(m-i) !(i !)^{2}}
$$


Any function, $y(x) \in L^{2}[0,1]$, can be approximated as a sum of shifted Legendre polynomials as

$$
y(x)=\sum_{i=0}^{\infty} c_{i} P_{i}(x)
$$

where

$$
c_{i}=\left\langle y(x), P_{i}(x)\right\rangle=(2 i+1) \int_{0}^{1} y(x) P_{i}(x) d x, \quad i=0,1, \ldots
$$

In general, the series in equation (2.4) can be truncated with the first $(N+1)$ shifted Legendre polynomials as

$$
y_{N}(x)=\sum_{i=0}^{N} c_{i} P_{i}(x)=C^{T} \varphi(x),
$$

where $C^{T}=\left[c_{0} c_{1} \cdots c_{N}\right]$ and $\varphi(x)=\left[P_{0}(x) P_{1}(x) \cdots P_{N}(x)\right]^{T}$.

The operational matrix of derivative of the shifted Legendre polynomials set $\varphi(x)$ is defined as [13]:

$$
\frac{d \varphi(x)}{d x}=D^{(1)} \varphi(x),
$$

where $D^{(1)}$ is the $(N+1) \times(N+1)$ operational matrix of derivative given as

$$
D^{(1)}=\left(d_{i j}\right)= \begin{cases}2(2 j-1) & \text { for } j=i-k \\ 0 & \text { otherwise }\end{cases}
$$

where

$$
k= \begin{cases}1,3, \ldots, N & \text { if } N \text { is odd } \\ 1,3, \ldots, N-1 & \text { if } N \text { is even }\end{cases}
$$

For example for even $N$ we have

$$
D^{(1)}=2\left(\begin{array}{cccccccc}
0 & 0 & 0 & 0 & \cdots & 0 & 0 & 0 \\
1 & 0 & 0 & 0 & \cdots & 0 & 0 & 0 \\
0 & 3 & 0 & 0 & \cdots & 0 & 0 & 0 \\
1 & 0 & 5 & 0 & \cdots & 0 & 0 & 0 \\
. & . & . & . & \cdots & . & . & . \\
1 & 0 & 5 & 0 & \cdots & 2 N-3 & 0 & 0 \\
0 & 3 & 0 & 7 & \cdots & 0 & 2 N-1 & 0
\end{array}\right) .
$$

From equation (2.7), it can be generalized for any $n \in N$ as

$$
\frac{d^{n} \varphi(x)}{d x^{n}}=\left(D^{(1)}\right)^{n} \varphi(x)=D^{(n)} \varphi(x), \quad n=1,2,3, \ldots
$$




\section{Application of the Method}

In this section, we derive the method for solving general second order linear and non-linear differential equations. Let us consider the general second order non-linear differential equation

$$
y^{\prime \prime}(x)+p(x) y^{\prime}(x)+f(x, y)=g(x), \quad \alpha, x \geq 0
$$

with initial conditions $y(0)=a$ and $y^{\prime}(0)=b$.

Approximating $y(x), p(x), f(x, y)$ and $g(x)$ by the shifted Legendre polynomials as

$$
\begin{gathered}
y(x) \approx \sum_{i=0}^{N} c_{i} P_{i}(x)=C^{T} \varphi(x), \\
p(x) \approx \sum_{i=0}^{N} p_{i} P_{i}(x)=P^{T} \varphi(x), \\
f(x, y) \approx f\left(x, C^{T} \varphi(x)\right), \\
g(x) \approx \sum_{i=0}^{N} g_{i} P_{i}(x)=G^{T} \varphi(x),
\end{gathered}
$$

where the unknowns are $C=\left[c_{0} c_{1} \cdots c_{N}\right]^{T}$.

Using Legendre operational matrix of differentiation, equation (1.6) can be written as

$$
C^{T} D^{2} \varphi(x)+P^{T} D^{1} \varphi(x)+f\left(x, C^{T} \varphi(x)\right) \approx G^{T} \varphi(x) .
$$

The residual $R_{N}(x)$ for equation (3.5) can be written as

$$
R_{N}(x) \approx C^{T} D^{2} \varphi(x)+P^{T} D^{1} \varphi(x)+f\left(x, C^{T} \varphi(x)\right)-G^{T} \varphi(x) .
$$

Applying typical Tau method, which is used in the sense of a particular form of the Petrov-Galerkin method [2], [10], [13], equation (3.5) can be transformed into $N-2$ nonlinear equations by applying

$$
\left\langle R_{N}(x), P_{i}(x)\right\rangle=\int_{0}^{1} R_{N}(x) P_{i}(x) d x=0, \quad i=0,1, \ldots, N-2 .
$$

The initial condition are given by

$$
y(0)=C^{T} \varphi(0)=d_{0} \quad \text { and } \quad y^{\prime}(0)=C^{T} D^{(1)} \varphi(0)=d_{1} .
$$

Equations (3.7) and (3.8) generate $N+1$ nonlinear algebraic equations. By solving these equations, unknowns in vector $C$ can be calculated. Thus, we can find the solution $y(x)$. 


\section{Results}

In this section, we apply the method presented in the previous section to solve some general second order nonlinear differential equations.

Example 4.1. We consider the differential equation

$$
y^{\prime \prime}(x)+\ln x y^{\prime}(x)+y=2+2 x \ln x+x^{2}
$$

with initial condition

$$
y(0)=0 \text { and } y^{\prime}(0)=0 .
$$

The exact solution of the problem is $y(x)=x^{2}$.

Now we apply our method for $N=2$ to illustrate the validity of our method.

For $N=2$, we have $y(x)=C^{T} \varphi(x)=c_{0} P_{0}(x)+c_{1} P_{1}(x)+c_{2} P_{2}(x)$ and given $p(x)=\ln x, f(x, y)=y$ and $g(x)=2+2 x \ln x+x^{2}$.

From equation (2.8), we have $D^{(1)}=\left(\begin{array}{lll}0 & 0 & 0 \\ 2 & 0 & 0 \\ 0 & 6 & 0\end{array}\right)$ and $D^{(2)}=\left(\begin{array}{ccc}0 & 0 & 0 \\ 0 & 0 & 0 \\ 12 & 0 & 0\end{array}\right)$ using equation (3.6), we get

$$
R_{2}(x)=C^{T} D^{(2)} \varphi(x)+\ln x C^{T} D^{(1)} \varphi(x)+C^{T} \varphi(x)-2-2 x \ln x-x^{2} .
$$

Therefore, using equation (3.7), we get

$$
c_{0}-2 c_{1}+15 c_{2}=\frac{11}{6},
$$

by applying initial conditions from equation (4.2), we have

$$
c_{0}-c_{1}+c_{2}=0 \text { and } 2 c_{1}-6 c_{2}=0 .
$$

Solving equations (4.4) to (4.5), we get

$$
c_{0}=\frac{1}{3}, \quad c_{1}=\frac{1}{2} \quad \text { and } \quad c_{2}=\frac{1}{6} .
$$

Hence the solution is obtained as

$$
\begin{aligned}
y(x) & =c_{0} P_{0}(x)+c_{1} P_{1}(x)+c_{2} P_{2}(x) \\
& =\left(\begin{array}{lll}
\frac{1}{3} & \frac{1}{2} & \frac{1}{6}
\end{array}\right)\left(\begin{array}{c}
1 \\
2 x-1 \\
6 x^{2}-6 x+1
\end{array}\right) \\
& =x^{2},
\end{aligned}
$$

which is the exact solution. That is by using only first three shifted Legendre polynimials, we have obtained the exact solution. 
Example 4.2. Taking $p(x)=\ln x, f(x, y)=y \cos x$ and $g(x)=2+$ $2 x \ln x+x^{2} \cos x$, equation (1.6) becomes

$$
y^{\prime \prime}+\ln x y^{\prime}(x)+y \cos x=2+2 x \ln x+x^{2} \cos x
$$

with initial condition

$$
y(0)=1 \quad \text { and } \quad y^{\prime}(0)=0 .
$$

The exact solution in this case is $y(x)=x^{2}$. Approximating $\cos x$ by 1 and taking $N=2$, we get the following algebraic equations

$$
\begin{gathered}
c_{0}-2 c_{1}+15 c_{2}=\frac{11}{6}, \\
c_{0}-c_{1}+c_{2}=0 \text { and } 2 c_{1}-6 c_{2}=0 .
\end{gathered}
$$

Solving equations from (4.8) to (4.9), we get

$$
c_{0}=\frac{1}{3}, \quad c_{1}=\frac{1}{2} \quad \text { and } \quad c_{2}=\frac{1}{6},
$$

and hence the solution is $y(x)=x^{2}$, which is the exact solution.

Example 4.3. Another particular example of nonlinear second order ordinary differential equation is

$$
y^{\prime \prime}(x)+\ln x y^{\prime}(x)+y^{2}=2+2 x \ln x+x^{4},
$$

with initial condition

$$
y(0)=0 \quad \text { and } \quad y^{\prime}(0)=0 .
$$

The exact solution of equation (4.10) is $y(x)=x^{2}$.

For $N=2$, we get the following three algebraic equations

$$
\begin{gathered}
c_{0}^{2}+\frac{1}{3} c_{1}^{2}+\frac{1}{5} c_{2}^{2}-2 c_{1}+15 c_{2}=\frac{17}{10}, \\
c_{0}-c_{1}+c_{2}=0 \quad \text { and } \quad 2 c_{1}-6 c_{2}=0 .
\end{gathered}
$$

Solving equations (4.12) to (4.13), we obtain

$$
c_{0}=\frac{1}{3}, \quad c_{1}=\frac{1}{2} \quad \text { and } \quad c_{2}=\frac{1}{6},
$$

and hence the solution is $y(x)=x^{2}$, which is again the exact solution. 
Example 4.4. Now, we consider the differential equation whose exact solution is in linear form, i.e., $y(x)=x$.

$$
y^{\prime \prime}(x)+e^{x} y^{\prime}(x)+e^{y}=2 e^{x},
$$

with initial condition

$$
y(0)=0 \text { and } y^{\prime}(0)=1 \text {. }
$$

For $N=1, p(x)=e^{x}, f(x, y)=e^{y}$ and $g(x)=2 e^{x}$, we obtain two equations by using equation (4.15) only, i.e.,

$$
c_{0}-c_{1}=0 \quad \text { and } \quad 2 c_{1}=1
$$

From equation (4.16), we get

$$
c_{0}=\frac{1}{2} \quad \text { and } \quad c_{1}=\frac{1}{2} .
$$

Hence the solution is $y(x)=x$, which is the exact solution.

Example 4.5. In this example, we consider the case when $p(x)=\sin x$, $f(x, y)=-y \cos x$ and $g(x)=-\cos x-1$, i.e.,

$$
y^{\prime \prime}(x)+\sin x y^{\prime}(x)-y \cos x=-\cos x-1
$$

with initial condition

$$
y(0)=1 \quad \text { and } \quad y^{\prime}(0)=0 .
$$

The exact solution of the problem is $y(x)=\cos x$. Approximating $\sin x$ with $x-\frac{x^{3}}{6}$ and $\cos x$ by $1-\frac{x^{2}}{2}$ and taking $N=2$, we get the following equations:

$$
\begin{gathered}
-\frac{5}{6} c_{0}+c_{1}+\frac{193}{15} c_{2}=-\frac{11}{6}, \\
c_{0}-c_{1}+c_{2}=1 \text { and } 2 c_{1}-6 c_{2}=0 .
\end{gathered}
$$

Solving simultaneously the equations (4.19) to (4.20), we have

$$
c_{0}=\frac{61}{71}, \quad c_{1}=-\frac{15}{71} \quad \text { and } \quad c_{2}=-\frac{5}{71},
$$

which gives the approximate solution of the differential equation (4.17) as $y(x)=1-\frac{30}{71} x^{2}$ and if we approximate $\sin x$ with $x$ and $\cos x$ by 1 , then the approximate solution becomes $y(x)=1-\frac{6}{14} x^{2}$. It is notable that by taking $N=3$ and approximating $\sin x$ with $x-\frac{x^{3}}{6}$ and $\cos x$ by $1-\frac{x^{2}}{2}$, we obtain better approximation to the solution as $y(x)=1-\frac{585}{1087} x^{2}+\frac{85}{1087} x^{3}$. So, one can observe that by increasing the value of $N$, we get more terms in the series solution and hence better approximation to the exact solution. 
Example 4.6. The series solution of the differential equation $y^{\prime \prime}(x)+$ $\frac{2}{x} y^{\prime}(x)+e^{x y^{2}}=x+1$ with initial conditions $y(0)=0$ and $y^{\prime}(0)=0$ is obtained in [11] as $y(x)=\frac{x^{3}}{12}-\frac{2}{15}\left(\frac{x^{3}}{12}\right)^{3}+\cdots$, and the solution obatined by applying our method taking $N=3$ and approximating $e^{x y^{2}}$ by 1 is $y(x)=\frac{x^{3}}{9}$.

Example 4.7. In the final example, we consider the differential equation

$$
y^{\prime \prime}(x)+e^{x} y^{\prime}(x)+e^{y}=2+2 x e^{x}+e^{x^{2}}
$$

with initial condition

$$
y(0)=0 \quad \text { and } \quad y^{\prime}(0)=0 .
$$

The exact solution of the problem is $y(x)=x^{2}$.

Now, by approximating $e^{y}$ with $1+y$ and for $N=2$, we get the following three algebraic equations.

$$
\begin{aligned}
& \frac{1}{2} c_{0}+\frac{13}{6} c_{1}+(12 e-24) c_{2}=\frac{5}{2} e-\frac{7}{2}, \\
& c_{0}-c_{1}+c_{2}=0 \text { and } 2 c_{1}-6 c_{2}=0 .
\end{aligned}
$$

Solving equations (4.23) to (4.24), we get

$$
c_{0}=2\left(\frac{5 e-7}{24 e-33}\right), \quad c_{1}=3\left(\frac{5 e-7}{24 e-33}\right) \quad \text { and } \quad c_{2}=\frac{5 e-7}{24 e-33} .
$$

Hence the solution is obtained as

$$
\begin{aligned}
y(x) & =c_{0} P_{0}(x)+c_{1} P_{1}(x)+c_{2} P_{2}(x) \\
& =\left(\begin{array}{lll}
2\left(\frac{5 e-7}{24 e-33}\right) & 3\left(\frac{5 e-7}{24 e-33}\right) & \frac{5 e-7}{24 e-33}
\end{array}\right)\left(\begin{array}{c}
1 \\
2 x-1 \\
6 x^{2}-6 x+1
\end{array}\right) \\
& =\frac{10 e-14}{8 e-11} x^{2},
\end{aligned}
$$

which is an approximate solution.

\section{Conclusion}

From the examples given in Section 3, we note that for different values of the functions $p(x), f(x, y)$ and $g(x)$, equation (1.6) can be solved by taking $N=2$, and using only first few shifted Legendre polynomials. In most of the cases, 
when the exact solution is in the form of a polynimial, we obtain the exact solution by applying our method. A good approximation to the exact solution is found otherwise. For the differential equations whose exact solution is a trigonometric function, we get better approximation by increasing the value of $N$, because with the increase in the value of $N$, we get more terms in the series solution.

\section{Acknowledgments}

This research was supported by the Science Research Foundation of Educational Department of Liaoning Province (L2012380).

\section{References}

[1] G. Adomian, R. Rach, Modified decomposition solution of linear and nonlinear boundary-value problems, Nonlinear Anal., 23 (1994), 615-619, doi: 10.1016/0362-546X(94)90240-2

[2] C. Canuto, M.Y. Hussaini, A. Quarteroni, T.A. Zang, Spectral Methods in Fluid Dynamic, Prentice-Hall, Englewood Cliffs, New Jersey, 1988.

[3] M.S.H. Chowdhury, I. Hashim, Solutions of a class of singular second-order IVPs by homotopy-perturbation method, Phys. Lett. A, 365 (2007), 439447, doi: $10.1016 /$ j.physleta.2007.02.002

[4] L. Cveticanin, The homotopy-perturbation method applied for solving complex-valued differential equations with strong cubic nonlinearity, $J$. Sound Vibration, 285 (2005), 1171-1179, doi: 10.1016/j.jsv.2004.10.026

[5] H.T. Davis, Introduction to Nonlinear Differential and Integral Equations, Dover, New York, 1962.

[6] J.H. He, X.H. Wu, Variational iteration method: New development and applications, Comput. Math. Appl., 54 (2007), 881-894, doi: 10.1016/j.camwa.2006.12.083

[7] M.M. Hosseini, H. Nasabzadeh, Modified Adomian decomposition method for specific second order ordinary differential equations, Appl. Math. Comput., 186 (2007), 117-123, doi: 10.1016/j.amc.2006.07.094 
[8] J.H. Lane, On theoretical temperature of the sun under the hypothesis of a gaseous mass maintaining its internal heat and depending on the laws of gases known to terrestrial experiment, Amer. J. Sci. Arts 2nd Series, 50 (1870), 57-74, doi: 10.2475/ajs.s2-50.148.57

[9] R.K. Pandey, N. Kumar, A. Bhardwaj, G. Dutta, Solution of Lane-Emden type equations using Legendre operational matrix of differentiation, Appl. Math. Comput., 218 (2012), 7629-7637, doi: 10.1016/j.amc.2012.01.032

[10] K. Parand, M. Razzaghi, Rational Chebyshev Tau method for solving higher-order ordinary differential equations, Int. J. Comput. Math., 81 (2004), 73-80.

[11] A. Rafiq, M. Ahmed, S. Hussain, A general approach to specific second order ordinary differential equations using homotopy perturbation method, Phys. Lett. A, 372 (2008), 4973-4976, doi: 10.1016/j.physleta.2008.05.070

[12] M.A. Rana, A.M. Siddiqui, Q.K. Ghori, R. Qamar, Application of He's homotopy perturbation method to sumudu transform, Int. J. Nonlinear Sci. Numer. Simul., 8 (2007), 185-190, doi: 10.1515/IJNSNS.2007.8.2.185

[13] A. Saadatmandi, M. Dehghan, A new operational matrix for solving fractional-order differential equations, Comput. Math. Appl., 59 (2010), 1326-1336, doi: 10.1016/j.camwa.2009.07.006

[14] A.M. Siddiqui, M. Ahmed, Q.K. Ghori, Couette and Poiseuille flows for non-newtonian fluids, Int. J. Nonlinear Sci. Numer. Simul., 7 (2006), 1526, doi: 10.1515/IJNSNS.2006.7.1.15

[15] A. Yildirim, T. Özis, Solutions of singular IVPs of Lane-Emden type by homotopy perturbation method, Phys. Lett. A, 369 (2007), 70-76, doi: 10.1016/j.physleta.2007.04.07 
